\title{
A Comparison Between Radioimmunotherapy and Hyperthermic Intraperitoneal Chemotherapy for the Treatment of Peritoneal Carcinomatosis of Colonic Origin in Rats
}

\author{
F. Aarts, MD, ${ }^{1}$ T. Hendriks, PhD, ${ }^{1}$ O. C. Boerman, $\mathrm{PhD},{ }^{2}$ M. J. Koppe, MD, PhD, ${ }^{1}$ \\ W. J. G. Oyen, MD, PhD, ${ }^{2}$ and R. P. Bleichrodt, $\mathrm{MD}, \mathrm{PhD}^{1}$
}

\footnotetext{
${ }^{1}$ Department of Surgery, Radboud University Nijmegen Medical Centre, PO Box 9101, 6500 HB, Nijmegen, The Netherlands ${ }^{2}$ Department of Nuclear Medicine, Radboud University Nijmegen Medical Centre, PO Box 9101, 6500 HB, Nijmegen, The Netherlands
}

Peritoneal carcinomatosis (PC) of colorectal cancer $(\mathrm{CRC})$ frequently is an end stage of colorectal cancer,

Received March 12, 2007; accepted June 12, 2007; published online: July 25, 2007.

Address correspondence and reprint requests to: F. Aarts, MD; E-mail: F.Aarts@chir.umcn.nl

Published by Springer Science+Business Media, LLC $\odot 2007$ The Society of Surgical Oncology, Inc. occurring in $5-50 \%$ of the patients, either synchronous or metachronous. ${ }^{1}$ If untreated, patients suffering from PC have a median survival of only 6 months. ${ }^{2}$ Survival is significantly improved by radical surgical debulking procedures (cytoreduction) in combination with intraperitoneal chemotherapy, either in combination with normothermia or hyperthermia (HIPEC). ${ }^{3-7}$ The median survival after cytoreductive surgery and HIPEC is $13-34$ months, ${ }^{4,8}$ 
and the 5-year survival rate is $19-27 \%$ at the cost of considerable morbidity and mortality rates of up to $23 \%$ and $4 \%$, respectively. ${ }^{6,9}$

In the latest reported clinical trial on adjuvant RIT in the setting of colon cancer, Liersch et al. reported results of a Phase II trial with ${ }^{131}$ I-labeled anti-CEA antibody labetuzumab administered to patients after complete resection of colorectal liver metastases. This study, where RIT was applied in an adjuvant setting to complete resection, resulted in a promising 5-year survival rate of $51.5 \% .{ }^{10}$ Radioimmunotherapy using radiolabeled monoclonal antibodies directed against tumor-associated antigens may therefore be an attractive anticancer therapy in patients with small volume disease.

We therefore have studied the application of RIT as adjuvant therapy following cytoreductive surgery (CS) in the setting of PC. In previous studies regarding PC of CRC in a rat model we showed that RIT could be an effective adjuvant treatment after CS. The efficacy of adjuvant RIT in combination with CS was investigated and compared with no treatment, CS only, and RIT only. The results of this study showed a significantly improved survival of animals treated with CS followed by RIT (median 88 days) compared with those treated with CS only (median 51 days) and RIT only (median 61.1 days). ${ }^{11}$ Based on the encouraging results, showing the observed increase in survival that was achieved with low-dose RIT and concomitant low toxicity, we now aimed to compare the efficacy of this treatment to that of today's standard of care, HIPEC, ${ }^{12,13}$ in a preclinical setting.

\section{MATERIALS AND METHODS}

\section{Animal Model of Peritoneal Carcinomatosis}

WAG/Rij rats (10-12 weeks old, body weight 240 290 g, Harlan Horst, The Netherlands) were housed under nonsterile standard conditions (temperature, $20-24^{\circ} \mathrm{C}$; relative humidity, 50-60\%; 12-h light/dark cycle) in filter-topped cages (two rats per cage), with free access to food (Ssniff, Bio Services Uden, The Netherlands) and water. Rats were accustomed to laboratory conditions for at least 1 week before experimental use. Peritoneal carcinomatosis was induced by intraperitoneal inoculation of $2.0 \times 10^{6} \mathrm{CC}$ 531 colon cancer cells, as described previously. ${ }^{14}$ All experiments were approved by the local Animal Welfare Committee of the Radboud University Nijmegen and were carried out in accordance with the Dutch Animal Welfare Act of 1997.

\section{Operative Procedure}

Prior to the laparotomy, all rats were given $10 \mathrm{~mL}$ of saline to prevent hypovolemia. Surgical procedures were performed under general anaesthesia using isoflurane $3 \%, \mathrm{O}_{2}$ and $\mathrm{N}_{2} \mathrm{O}$ 1:1. Thirty minutes prior to and once daily until the third day postoperatively, rats were given buprenorphine $(5 \mu \mathrm{g}, 0.1 \mathrm{~mL} / \mathrm{rat} /$ day $)$ for analgesia. During the operation, rats were placed on a warmed mattress to limit body heat loss. All rats underwent a midline laparotomy. After opening the abdomen the extent of intraperitoneal tumor growth was scored semiquantitatively, 0 (no macroscopic tumor), 1 (little; located at 1-2 sites with a diameter of 1-2 mm), 2 (moderate; located at 1-2 sites and a diameter $2-5 \mathrm{~mm}$ ), or 3 (abundant; located at multiple sites and/or diameter $>5 \mathrm{~mm}$ ) in all four quadrants of the abdomen. The sum of the tumor scores of all sites represented the peritoneal cancer index $(\mathrm{PCI}){ }^{11}$

Subsequently, CS, including a routine omentectomy, was performed in all rats. Irresectable tumor deposits were cauterized using an electrocoagulation device. After completion of the surgical cytoreduction, the abdominal wall was closed in two layers using continuous Vicryl 3/0 sutures for the muscular component and iron wound clips for the skin in animal treated with CS only or CS + RIT.

\section{Monoclonal Antibody, Radiolabeling, and RIT}

The murine MG1 monocolonal antibody (MAb), an anti-CC531 IgG2a monoclonal antibody that recognizes a $80 \mathrm{kDa}$ cell surface antigen and localizes preferentially in tumors when injected in rats bearing CC-531 tumors, ${ }^{15}$ was purchased from Antibodies for Research Applications BV (Gouda, The Netherlands). Labeling of the antibody with ${ }^{177} \mathrm{Lu}$ was carried out as previously described. ${ }^{11}$ In brief, the $\mathrm{MAb}$ was conjugated with 2-(4-isothiocyanatobenzyl)-diethylenetriaminepenta-acetic acid (ITCDTPA) (Macrocyclics, Dallas, TX) and subsequently labeled with ${ }^{177}$ Lutetium (IDB Holland, Baarle Nassau The Netherlands) and purified by gel filtration on a PD10 column (Amersham, Pharmacia Biotech, Maarsen, The Netherlands). The purified ${ }^{177} \mathrm{Lu}-\mathrm{MG} 1$ preparation was diluted in PBS with $0.5 \%$ BSA for injection, the specific activity of the administered ${ }^{177} \mathrm{Lu}-\mathrm{MG} 1$ preparation was $0.4 \mathrm{MBq} /$ $\mu \mathrm{g}$. The labeling procedure using ${ }^{177} \mathrm{Lu}$ was performed under strict metal-free conditions.

RIT (185 $\mu \mathrm{g}$ MG1/ rat, radiolabeled with $74 \mathrm{MBq}$ ${ }^{177} \mathrm{Lu}$ in $3.0 \mathrm{~mL}$ ) was intraperitoneally injected 
immediately after surgery, as this was determined to be the most optimal time for adjuvant administration. ${ }^{16}$

\section{Mitomycin-C}

Mitomycin-C (MMC) was obtained from $\mathrm{Ny}-$ comed Christiaens BV (Breda, The Netherlands) as a powder in glass vial $(40 \mathrm{mg} / \mathrm{vial})$. Immediately before use, MMC was dissolved in $0.9 \%$ sodium chloride to the appropriate concentrations.

\section{HIPEC Procedure}

Following CS, while the abdomen was still exposed, two multiperforated catheters (Argyle, Sherwood Medical, Ireland) were inserted laterally through the abdominal wall and subsequently fixed in the abdominal cavity. The inflow drain was placed in the right paracolic gutter, the outflow drain in the left subdiaphragmatic space. The intraperitoneal temperature was monitored with an intra-abdominal thermometer (PTFE Insulated thermocouple, VWR International, Amsterdam, The Netherlands), at the site with generally the highest tumor load (omentum). In addition, a thermometer was placed inside the rectum. After placement of the catheters, the abdominal wall was closed using a continuous suture (Ethilon 3.0, Johnson \& Johnson, Ethicon) (Fig. 1). During the HIPEC procedure, rats were removed from the warmed mattress to prevent general hyperthermia. The perfusion system was filled with $250 \mathrm{~mL}$ saline, containing $4 \mathrm{mg}$ MMC (Mitomycin-C Kyowa, Christiaens). The perfusate was heated in a tube coil using a thermostatically regulated water bath set to a temperature of $48^{\circ} \mathrm{C}$ and infused into the peritoneal cavity by a roller pump (Ismatec IPS-8, Ismatec SA, Glattbrugg, Switzerland) for the duration of 60 minutes at $10 \mathrm{~mL} / \mathrm{min}$. Abdominal inflow temperature was set at $44^{\circ} \mathrm{C}$. In order to achieve a uniform heat distribution, gentle massage of the abdomen was applied throughout the duration of the HIPEC procedure. After completion of the perfusion, the abdominal cavity was flushed with warmed $\left(37^{\circ} \mathrm{C}\right)$ saline for a period of 10 minutes. The abdomen was opened again to remove the catheters. Subsequently, the abdomen was closed as described previously.

\section{Intraperitoneal Distribution of MMC and Dose Determination}

Prior to the therapy experiment with HIPEC, we investigated the intraperitoneal distribution of the perfusion fluid using a methylene blue stained per-

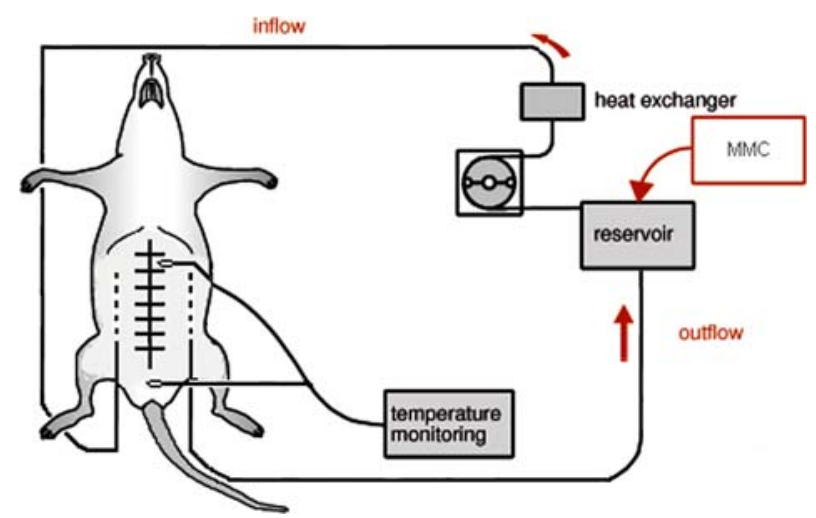

FIG. 1. HIPEC Perfusion System; MMC Mitomycin C Kyowa 16 $\mathrm{mg} / \mathrm{L}$ perfusate. Adapted from Ref. 19. Reproduced with permission.

fusate. The perfusate was administered in the same fashion as in the therapeutic experiment. After completion, the abdominal cavity was inspected for the presence of blue dye in all quadrants on both parietal as well as visceral peritoneum of the intra-abdominal organs and the diaphragm. Subsequently, a study to determine the dose of MMC that resulted in acceptable toxicity was performed in nine animals (three animals per group). Animals underwent a laparotomy including an omentectomy and complete bowel inspection followed by heated perfusion of the abdominal cavity with MMC at $4 \mathrm{mg} / \mathrm{L}$ or $16 \mathrm{mg} / \mathrm{L}$. Control rats underwent laparotomy and an omentectomy only. Body weight and physical condition were monitored during 6 days following the procedure to assess treatment-related toxicity.

\section{Treatment Efficacy}

Seven days after intraperitoneal tumor induction with $2.0 \times 10^{6}$ CC-531 tumor cells, 45 rats, 15 per treatment group, were randomly assigned to undergo either CS only, CS + RIT or CS + HIPEC. The operative procedures and application of the adjuvant therapies were performed as described previously. Toxicity of the treatment was determined clinically and by weighing the rats. Body weight was expressed as relative body weight compared with the body weight on the day of surgery. Survival was scored, and at autopsy the extent of tumor growth was determined.

\section{Follow-Up}

The primary endpoint was 16 -week survival. As part of monitoring the physical condition during the 
immediate postoperative period, the general condition was monitored and the body weight was measured daily during the first 2 weeks. When the humane endpoint (HEP) was reached (signs of massive hemorrhagic ascites, physical inactivity or signs of intra-abdominal tumor growth with invalidating consequences), rats were killed by $\mathrm{O}_{2} / \mathrm{CO}_{2}$-administration and immediately dissected. The HEP was determined by an experienced biotechnician who was blinded to the therapeutic regimen. At the time of the HEP rats were generally lethargic, showing signs of advanced PC as the presence of ascites. At dissection, the intraperitoneal tumor growth was scored as described previously. At 16 weeks postoperatively, the study was terminated and the remaining rats were euthanized and dissected. In case of absence of macroscopic tumor, all relevant organs, including the greater momentum, the mesentery, and the diaphragm were removed for histopathological staining to determine tumor presence microscopically. Sections were stained using hematoxylin \& eosin (H\&E) and/or immunohistochemical staining using the murine MG1 antibody in combination with a horseanti-mouse IgG antibody, HRP conjugated (Vector Laboratories Inc., Burlingame, CA, USA).

\section{Statistical Analysis}

Statistical analysis was performed using SPSS (Chicago, IL) software and Graphpad Prism (Graphpad Software Inc., San Diego CA) for analysis. Comparison of dichotomous values was done using chisquare or Fisher's Exact test. Nonparametric testing was performed using two-way ANOVA testing. Survival curves were analyzed using Kaplan-Meier curves and compared by means of the log-rank test. Posttesting using Bonferroni was applied to correct for multiple groups. All tests were two-sided; the level of statistical significance was set at a $P$ value of $<.05$.

\section{RESULTS}

\section{Intraperitoneal Distribution of HIPEC and Dose Determination}

The intraperitoneal distribution of the perfusate administered according to the previously described procedure, showed a distribution pattern amongst all quadrants, including the diaphragm bilaterally and at the mesenterial root (Fig. 2).

The applied dose of $16 \mathrm{mg} \mathrm{MMC/L}$ resulted in a maximum mean weight loss of $13.7 \pm 2.9 \%$ at 4

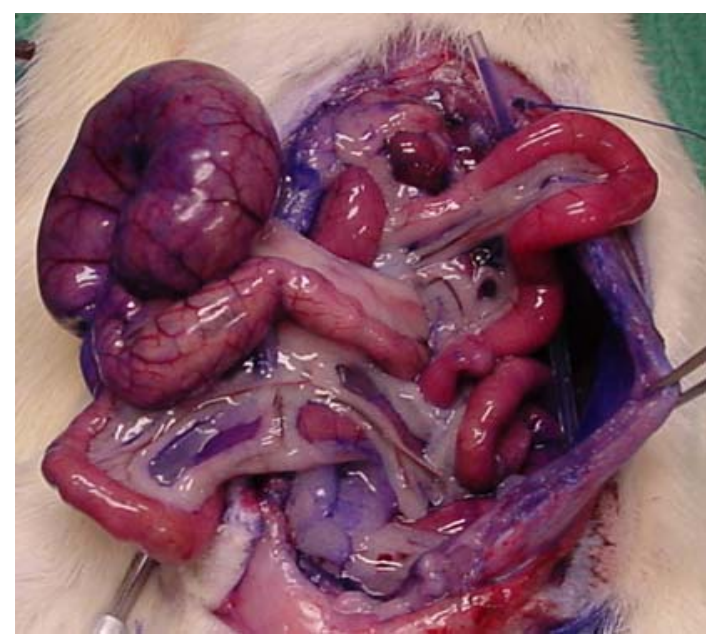

FIG. 2. Intraperitoneal distribution of methylene blue stained perfusate.

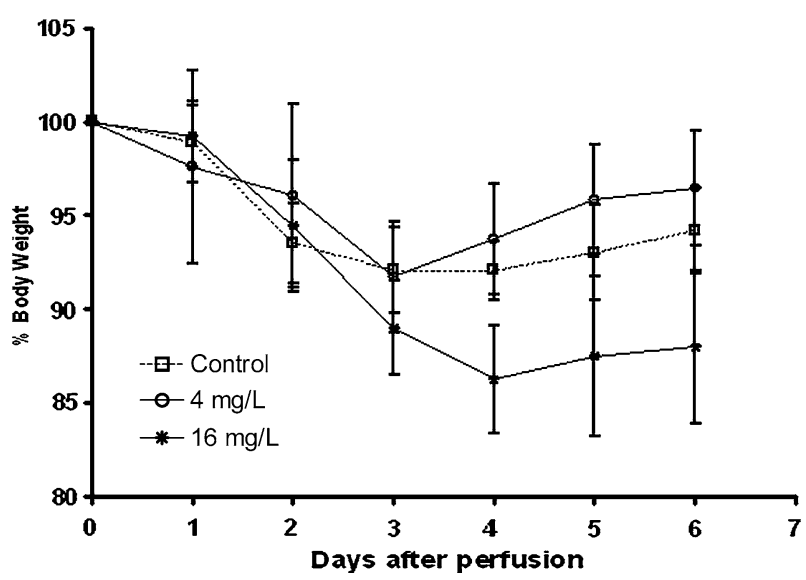

FIG. 3. The relative body weight of Wag/Rij rats after exploratory laparotomy (control) and heated intraperitoneal chemotherapy (HIPEC) given immediately postoperatively in different doses. Data represent means \pm standard error of the mean (SEM).

days postoperatively. In addition, the first 3 days following the heated perfusion, animals were lethargic and suffered from diarrhea from day 2 until day 4 postoperatively. In contrast, the maximum weight loss in the $4 \mathrm{mg} / \mathrm{L}$ was $8.3 \pm 2.9 \%$ at day 3 and $7.5 \pm 2.3 \%$ at day 3 in the control group (Fig. 3). None of the animals died during the immediate postoperative period. Based on these observations, HIPEC, when administered at a dose of $16 \mathrm{mg} / \mathrm{L}$ for the duration of 60 minutes at the given temperature, was considered to be the maximal tolerable dose to be used for the HIPEC procedure. 
TABLE 1. Treatment group characteristics

\begin{tabular}{|c|c|c|c|}
\hline & \multicolumn{3}{|l|}{ PCI } \\
\hline & \multicolumn{3}{|l|}{ Median (range) } \\
\hline & $\mathrm{CS}$ & $\mathrm{CS}+\mathrm{HIPEC}$ & $\mathrm{CS}+\mathrm{RIT}$ \\
\hline Preoperative body weight (g) & $266(251-287)$ & $264(245-285)$ & $262(244-276)$ \\
\hline \multicolumn{4}{|l|}{ Tumor score per site } \\
\hline Greater omentum & $2(2-3)$ & $2(1-2)$ & $2(1-2)$ \\
\hline Liver hilum & $1(0-1)$ & $1(0-1)$ & $1(0-1)$ \\
\hline Perisplenic & $0(0-1)$ & $0(0)$ & $0(0)$ \\
\hline Mesentery & $1(0-2)$ & $1(0-2)$ & $1(0-2)$ \\
\hline Gonadal fatpads & $0(0-2)$ & $0(0-2)$ & $1(0-2)$ \\
\hline Diaphragm & $0(0-1)$ & $0(1)$ & $0(0-1)$ \\
\hline Parietal peritoneum & $1(0-1)$ & $1(0-1)$ & $1(0-1)$ \\
\hline Total & $5(4-8)$ & $5(4-6)$ & $5(4-8)$ \\
\hline \multicolumn{4}{|c|}{ Resection macroscopically complete } \\
\hline Yes & 12 & 13 & 13 \\
\hline No & 3 & 2 & 2 \\
\hline
\end{tabular}

Treatment group characteristics (peritoneal cancer index; PCI) found during laparotomy before the administration of the adjuvant therapy. CS, cytoreductive surgery; HIPEC, heated intraperitoneal chemotherapy; RIT, radioimmunotherapy. PCI is expressed as median and range.

\section{Operative Procedure}

Preoperative body weight did not differ between groups, $P=.52$ (Table 1) At laparotomy, tumor nodules were present in the omentum, liver hilum, the mesentery, and gonadal fatpads ( $1-3 \mathrm{~mm}$ diameter). Median PCI score at time of surgery was 5 (range 48) and was similar in all experimental groups. After surgical cytoreduction, residual disease remained in situ in 7 rats after cauterization and was equally distributed among the groups $(P=.84)$. The surgical procedures without adjuvant therapy took 20-30 minutes per animal.

There was no intraoperative mortality. However, one rat in the CS + HIPEC and one rat in the CS + RIT group were euthanized after 2 and 9 days, respectively. The animal in the CS + HIPEC group showed massive weight loss as a result of bowel necrosis and subsequent perforation, the cause of death of the animal in the CS + RIT group remained unclear. The median intra-abdominal temperature during the HIPEC procedure, measured at the site where the greater omentum was removed, was $41.0^{\circ} \mathrm{C}$ (range $40.4-41.6^{\circ} \mathrm{C}$ ). In contrast, the median rectal temperature was $34.6^{\circ} \mathrm{C}$ (range $34.1-34.8^{\circ} \mathrm{C}$ ) (Fig. 4).

CS and CS + RIT were well tolerated, whereas animals in the CS + HIPEC groups showed signs of physical discomfort; animals of the latter group were generally lethargic and showed pilo erection two days following the procedure. In addition, these animals all suffered from diarrhea up to 4 days after the HIPEC procedure. The relative body weight after of the various treatment groups is depicted in Fig. 5. Maximum body weight loss after CS or CS + RIT

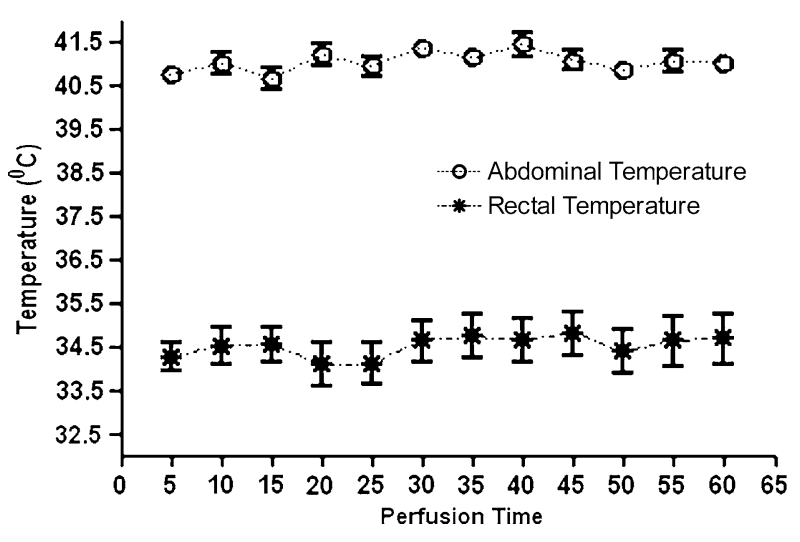

FIG. 4. The recorded intra-abdominal and rectal temperature during the HIPEC procedure. Data represent means \pm standard error of the mean (SEM).

was similar $(7.3 \pm 2.6 \%$ vs $9.3 \pm 1.8 \% 4$ days postoperatively, $P>.05)$. Rats that received adjuvant HIPEC had a maximum body weight loss of $12.3 \pm 1.7 \%$, which was significantly higher than that after CS alone $(P<.001)$ or CS + RIT $(P<$ $.001)$. Rats generally gained weight from the fifth postoperative day onward. In the HIPEC group, however, postoperative mean body weight remained significantly lower than that of the animals in the CS group, until 5 weeks postoperatively.

\section{Treatment Efficacy}

During the experiment, 29 animals were euthanized because of massive amounts of ascites that resulted from intraperitoneal tumor growth. The mean 


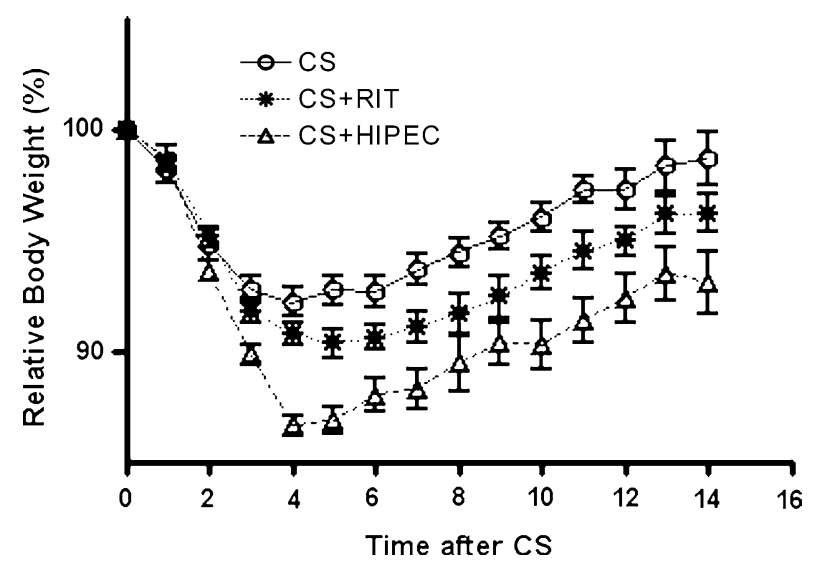

FIG. 5. The relative body weight of Wag/Rij rats with small peritoneal CC-531 tumors in the first 14 days after cytoreductive surgery (CS) only, CS + radioimmunotherapy given immediately postoperatively (RIT) or heated intraperitoneal chemotherapy (HIPEC) given immediately postoperatively. Data represent means \pm standard error of the mean (SEM).

amount of ascites at the humane endpoint was $31 \pm 22.6 \mathrm{~mL}, 26.5 \pm 23.8 \mathrm{~mL}$, and $26.4 \pm 22.6$ $\mathrm{mL}$ in the CS, RIT, and HIPEC groups, respectively $(P=.82)$. At the time of death, mean PCI in the CS, CS + HIPEC, and CS + RIT groups was 18 (range 9-22), 12 (range 5-15), and 18 (range 16-19), respectively, with significant differences between the $\mathrm{CS}+$ HIPEC and both other treatment groups $(P<$ .001 for both comparisons).

The survival curves of the various treatment groups are depicted in Fig. 6. Median survival of the rats that were treated with CS only was 57 days (range 36-112). Adjuvant HIPEC resulted in a median survival of 76 days (range 33-112), $P=.17$, when compared with CS only. Median survival of the rats that were treated with CS followed by the adjuvant administration of RIT was improved to a median survival of 97 days (range 49-112), $P<.001$ compared with CS only. When compared with CS followed by adjuvant HIPEC, the adjuvant administration of RIT to surgery did not result in an improved survival $(P=.33)$.

At the endpoint of the study, 16 weeks after CS, 14 animals (two animals in the CS group, five animals in the CS + HIPEC group, and seven animals in the CS + RIT group) were still alive, without any physical signs of intraperitoneal tumor growth.

Of these 14 animals that were still alive 16 weeks after surgery, one animal in the CS + HIPEC group and three animals in the CS + RIT group showed macroscopic evidence of tumor at dissection. In the remaining 10 animals (two in the CS alone group,

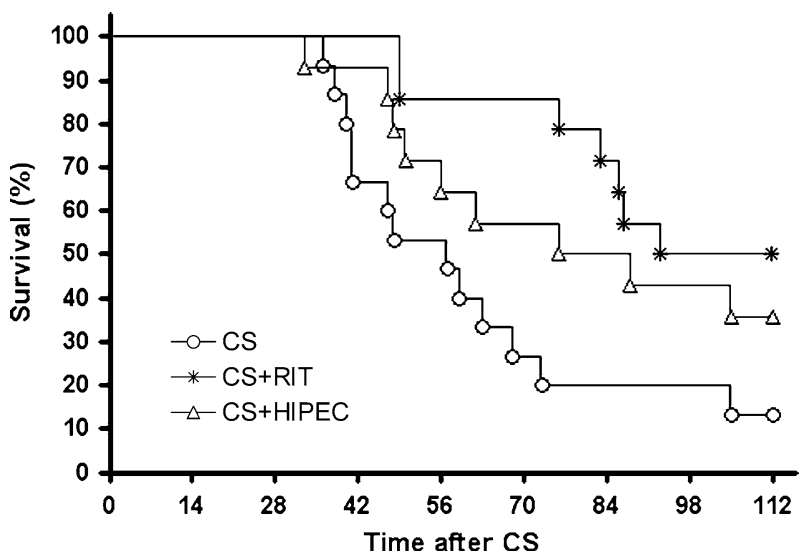

FIG. 6. Kaplan-Meier survival curves for Wag/Rij rats with small peritoneal CC-531 tumors after cytoreductive surgery (CS), CS + RIT (RIT) or CS + HIPEC (HIPEC).

four in the HIPEC group, and four in the RIT group) not even microscopic tumor presence was found.

\section{DISCUSSION}

In the present study, adjuvant radioimmunotherapy after cytoreductive surgery for peritoneal carcinomatosis of colorectal origin in rats significantly improved survival, whereas HIPEC did not. In addition, the application of HIPEC was associated with considerably more toxicity as compared to RIT.

The treatment of peritoneal carcinomatosis was studied with CC-531 syngeneic tumors that grew intraperitoneally in Wag/Rij rats. This model is highly reproducible, and the growth and distribution pattern throughout the abdominal cavity are similar to the human entity of PC. ${ }^{14}$ Cytoreduction performed at 7 days after tumor reduction resulted in minimal residual disease $(<1 \mathrm{~mm})$. In this setting, both HIPEC and RIT result in maximum therapeutic efficacy. ${ }^{12,13,17}$

The MG1 MAb preferentially localizes in the CC531 tumors, ${ }^{11}$ with only minor localization in thymus, lymph node, salivary gland tissue, and skin. ${ }^{15}{ }^{177} \mathrm{Lu}$ was selected as the radionuclide for RIT of minimal residual disease because of its high tumor uptake and adequate physical properties including a medium-energy $\beta$-emission with a maximum penetration range in tissue of $2.5 \mathrm{~mm}$. In our previous studies we have used the combination of ${ }^{177} \mathrm{Lu}-\mathrm{MG} 1$ radionuclide-antibody. These studies demonstrated the combination to be highly effective for the improvement of survival in the model of PC as described previously. ${ }^{11,16}$ Moreover, in previous experiments we have shown that 
radioimmunotherapy with a radiolabeled irrelevant antibody is less effective by far compared with therapy with a radiolabeled specific antibody. ${ }^{18}$ HIPEC has been studied in only a few preclinical studies. ${ }^{19-22}$ These studies showed that its use was associated with a decreased tumor load compared with control groups. ${ }^{19}$ However, in these studies, HIPEC was associated with a considerable toxicity, indicated by lethargy, marked body weight loss, and bacterial translocation. ${ }^{23}$ These results on toxicity are in corroboration with the results of our study and mimic the clinical effects of HIPEC. Clinical studies with postoperative intraperitoneal chemotherapy are associated with a high mortality and morbidity. ${ }^{12}$

Of 16 published reports on the use of HIPEC in the clinical setting, 13 reports described the administration of MMC. ${ }^{12}$ In vitro, MMC has also shown to inhibit growth of CC531 cells in a concentration a thousand-fold lower than the concentration used in the present experiment. ${ }^{24}$ The applied dose of $16 \mathrm{mg} /$ L MMC in our study is within the range of doses applied in clinical practice $\left(5-20 \mathrm{mg} / \mathrm{L}^{25}\right)$ and is higher than described in other preclinical studies $\left(2.25 \mathrm{mg} / \mathrm{L}^{19}\right.$ and $\left.4 \mathrm{mg} / \mathrm{L}^{22}\right)$. In addition, in the present study HIPEC was applied as an adjuvant treatment to cytoreduction, whereas Pelz et al. ${ }^{19}$ applied HIPEC as monotherapy with subsequent killing of the animals after only 10 days.

The intraperitoneal distribution of MMC during the perfusion was studied before the start of the actual experiment and showed equal distribution among all quadrants. The effect of the perfusion technique on distribution differences and their associated differences in survival have never been studied clinically. Glehen and colleagues performed a large clinical study in 506 patients that were treated with both the open and closed abdomen perfusion technique. The authors reported no differences in survival between both perfusion techniques. ${ }^{6}$ This observation was confirmed by the study of Sugarbaker and colleagues. ${ }^{26}$

RIT with $74 \mathrm{MBq}$ of the ${ }^{177} \mathrm{Lu}-\mathrm{MG} 1$ radionuclideantibody in $3 \mathrm{~mL}$ has previously been shown to be an effective treatment for experimentally induced PC of colonic origin when administered intraperitoneally. ${ }^{11,16}$ The biodistribution of intraperitoneally injected ${ }^{111}$ In-labeled MG1 was studied by Koppe et al. and showed a preferential uptake of the radiolabeled antibody in the tumor. ${ }^{11}$

The extraperitoneal temperature of $48^{\circ} \mathrm{C}$ necessary to obtain inflow temperatures of $44^{\circ} \mathrm{C}$ would not have had a negative influence on the cytotoxicity of MMC, since Ahrar et al. ${ }^{27}$ showed that only temperatures exceeding $60^{\circ} \mathrm{C}$ decreased its cytotoxic ef- fect. On the other hand, one has to bear in mind the fact that the additive effect of hyperthermia in HIPEC in the clinical setting has not yet been proven in a randomized trial. Elias et al. and Glehen et al. both reported the use of early postoperative chemotherapy (EPIC), without hyperthermia, ranging from day 1 to day 5 after surgery, and HIPEC. Elias et al. found no significant difference in survival. Similarly, in the multicenter study in 506 patients of whom 53.3 and $24.3 \%$ underwent HIPEC and EPIC alone, no significant difference was found in survival between the two treatment groups. ${ }^{6}$ The duration of the heated perfusion, $60 \mathrm{~min}$. in this experiment, is in concordance with the recent consensus statement regarding Cytoreductive Surgery and Hyperthermic Intraperitoneal Chemotherapy in the Management of Peritoneal Surface Malignancies of Colonic Origin, stating that the perfusion should last $60-120$ minutes. $^{13}$ These data on the dose of MMC, the used perfusion time and temperature, together with our results that showed antitumor effect in the model of induced PC from CC531 cells (significantly lower PCI at HEP in favor of CS + HIPEC), we can conclude that the HIPEC model used in our study was able to induce regression of $\mathrm{PC}$ of colonic origin.

In the latest reported clinical trial on adjuvant RIT in the setting of colon cancer, Liersch et al. reported results of a Phase II trial with ${ }^{131}$ I-labeled anti-CEA antibody labetuzumab administered to patients after complete resection of colorectal liver metastases. This study, where RIT was applied in an adjuvant setting to complete resection, resulted in a promising 5-year survival rate of $51.5 \% .^{10}$ The results of this study is in accordance with the conclusion of a recent review on the use of RIT to treat colon cancer. ${ }^{17}$ In this review, the authors state that the time may have come for clinical trials in which RIT is added to standard regimens to establish the place of this treatment modality as an adjuvant treatment after CS.

To our knowledge, the present study is the first study comparing the use of RIT and HIPEC in an adjuvant setting to $\mathrm{CS}$ for the treatment of $\mathrm{PC}$ in colorectal cancer. Our preclinical studies indicate that the application of RIT immediately following CS can improve survival in rats with PC of CRC. Moreover, from the present study we conclude that the use of adjuvant RIT is an effective treatment with low toxicity. When compared with today's standard of care, HIPEC, RIT was at least as effective. RIT consisted of an activity dose of $74 \mathrm{MBq}$ of ${ }^{177} \mathrm{Lu}$-labeled MG1per rat, resulting in only minor toxicity, whereas the theoretical MTD of ${ }^{177} \mathrm{Lu}$-labeled antibodies in $250 \mathrm{~g}$ rats could be approximately $150 \mathrm{MBq} .{ }^{11}$ 
There are, however, some challenges to the clinical applications of adjuvant RIT. For example, after cytoreductive surgery, patients are transferred to the intensive care unit. Optimal patient care has to be balanced with radiation safety issues for the medical staff. Previously, we reported on the optimal time interval between RIT and CS. ${ }^{16}$ In that study, we showed that RIT should be administered as soon as possible after CS, with a window of opportunity for RIT administration up to 4 days after surgery. It can therefore be envisioned that for radiation safety reasons the therapy should be given not before discharge of the patient from ICU and removal of the abdominal drains.

Our study therefore justifies the consideration of intraperitoneal radioimmunotherapy after cytoreductive surgery in case of peritoneal carcinomatosis of colorectal cancer. In clinical studies, this approach should be compared with HIPEC.

\section{CONCLUSION}

This study showed that RIT adjuvant to CS significantly improved survival compared to CS alone in a rat model of PC of CRC, whereas the contemporary gold standard, HIPEC, did not cause a significant improvement in survival. This improvement of survival was associated with a decreased level of treatment-related toxicity compared with HIPEC. Adjuvant radioimmunotherapy might therefore be an alternative adjuvant treatment after cytoreductive surgery of PC of colorectal origin in a clinical trial setting.

\section{ACKNOWLEDGMENT}

The authors wish to thank Ben de Man and Roger Lomme (Radboud University Nijmegen Medical Centre, Surgical Research Laboratory) for their excellent assistance during the operative procedures, and Gerry Grutters, Henny Eikholt and Bianca Lemmers (Radboud University Nijmegen Medical Centre, Central Animal Laboratory) for their assistance in the animal experiments.

\section{REFERENCES}

1. Sugarbaker PH, Cunliffe WJ, Belliveau J, et al. Rationale for integrating early postoperative intraperitoneal chemotherapy into the surgical treatment of gastrointestinal cancer. Semin Oncol 1989; 16:83-97.
2. Jayne DG, Fook S, Loi C, et al. Peritoneal carcinomatosis from colorectal cancer. Br J Surg 2002; 89:1545-50.

3. Sugarbaker PH. Colorectal carcinomatosis: a new oncologic frontier. Curr Opin Oncol 2005; 17:397-9.

4. Culliford AT, Brooks AD, Sharma S, et al. Surgical debulking and intraperitoneal chemotherapy for established peritoneal metastases from colon and appendix cancer. Ann Surg Oncol 2001; 8:787-95.

5. Stephens AD, Alderman R, Chang D, et al. Morbidity and mortality analysis of 200 treatments with cytoreductive surgery and hyperthermic intraoperative intraperitoneal chemotherapy using the coliseum technique. Ann Surg Oncol 1999; 6:790-6.

6. Glehen O, Kwiatkowski F, Sugarbaker PH, et al. Cytoreductive surgery combined with perioperative intraperitoneal chemotherapy for the management of peritoneal carcinomatosis from colorectal cancer: a multi-institutional study. J Clin Oncol 2004; 22:3284-92.

7. Verwaal VJ, van RS, de BE, et al. Randomized trial of cytoreduction and hyperthermic intraperitoneal chemotherapy versus systemic chemotherapy and palliative surgery in patients with peritoneal carcinomatosis of colorectal cancer. J Clin Oncol 2003; 21:3737-43.

8. Glehen O, Cotte E, Schreiber V, et al. Intraperitoneal chemohyperthermia and attempted cytoreductive surgery in patients with peritoneal carcinomatosis of colorectal origin. $\mathrm{Br}$ $J$ Surg 2004; 91:747-754.

9. Elias D, Blot F, El OA, et al. Curative treatment of peritoneal carcinomatosis arising from colorectal cancer by complete resection and intraperitoneal chemotherapy. Cancer 2001; 92:71-76.

10. Liersch T, Meller J, Kulle B, et al. Phase II trial of carcinoembryonic antigen radioimmunotherapy with 131I-labetuzumab after salvage resection of colorectal metastases in the liver: five-year safety and efficacy results. J Clin Oncol 2005; 23:6763-70.

11. Koppe MJ, Hendriks T, Boerman OC, et al. Radioimmunotherapy is an effective adjuvant treatment modality after cytoreductive surgery of peritoneal carcinomatosis of colonic origin. J Nucl Med 2006; 47:1867-74.

12. Koppe MJ, Boerman OC, Oyen WJ, et al. Peritoneal carcinomatosis of colorectal origin: incidence and current treatment strategies. Ann Surg 2006; 243:212-22.

13. Esquivel J, Sticca R, Sugarbaker P, et al. Cytoreductive Surgery and Hyperthermic Intraperitoneal Chemotherapy in the Management of Peritoneal Surface Malignancies of Colonic Origin: A Consensus Statement. Ann Surg Oncol 2006.

14. Lopes Cardozo AM, Gupta A, Koppe MJ, et al. Metastatic pattern of CC531 colon carcinoma cells in the abdominal cavity: an experimental model of peritoneal carcinomatosis in rats. Eur J Surg Oncol 2001; 27:359-63.

15. Hagenaars M, Koelemij R, Ensink NG, et al. The development of novel mouse monoclonal antibodies against the CC531 rat colon adenocarcinoma. Clin Exp Metastasis 2000; 18:281-9.

16. Aarts F, Koppe MJ, Hendriks T, et al. Timing of adjuvant radioimmunotherapy after cytoreductive surgery in experimental peritoneal carcinomatosis of colorectal origin. Ann Surg Oncol 2007; 14:533-40.

17. Koppe MJ. Radioimmunotherapy and colorectal cancer. $\mathrm{Br} J$ Surg 2005; 92:264-76.

18. Koppe MJ, Soede AC, Pels W, et al. Experimental radioimmunotherapy of small peritoneal metastases of colorectal origin. Int J Cancer 2003; 106:965-72.

19. Pelz JO, Doerfer J, Hohenberger W, et al. A new survival model for hyperthermic intraperitoneal chemotherapy (HIPEC) in tumor-bearing rats in the treatment of peritoneal carcinomatosis. BMC Cancer 2005; 5:56.

20. Zeamari S, Floot B, van d V, et al. Pharmacokinetics and pharmacodynamics of cisplatin after intraoperative hyperthermic intraperitoneal chemoperfusion (HIPEC). Anticancer Res 2003; 23:1643-8. 
21. Pestieau SR, Belliveau JF, Griffin H, et al. Pharmacokinetics of intraperitoneal oxaliplatin: experimental studies. J Surg Oncol 2001; 76:106-14.

22. Makrin V, Lev-Chelouche D, Even SE, et al. Intraperitoneal heated chemotherapy affects healing of experimental colonic anastomosis: an animal study. J Surg Oncol 2005; 89:18-22.

23. Bozer M, Turkcapar N, Bayar S, et al. Intraperitoneal hyperthermic perfusion may induce bacterial translocation. Hepatogastroenterology 2005; 52:111-4.

24. Dirix LY, Gheuens EE, van der HS, et al. Cytotoxic activity of 7-N-(2-((2-(-gamma-L-glutamylamino)- ethyl)dithio)ethyl)mitomycin $\mathrm{C}$ and metabolites in cell lines with different resistance patterns. Anticancer Drugs 1994; 5:343-54.
25. van Ruth S, Verwaal VJ, Zoetmulder FA. Pharmacokinetics of intraperitoneal mitomycin C. Surg Oncol Clin N Am 2003; 12:771-780.

26. Sugarbaker PH, Stuart OA, Yoo D. Strategies for management of the peritoneal surface component of cancer: cytoreductive surgery plus perioperative intraperitoneal chemotherapy. $J$ Oncol Pharm Pract 2005; 11:111-9.

27. Ahrar K, Newman RA, Pang J, et al. 2004 Dr. Gary J. Becker Young Investigator Award: Relative thermosensitivity of cytotoxic drugs used in transcatheter arterial chemoembolization. J Vasc Interv Radiol 2004; 15:901-5. 